The effect of inter-stimulus interval on sequential effects in absolute identification

William J. Matthews

and

Neil Stewart

University of Warwick

RUNNING HEAD: Sequential effects

Address correspondence to:

William Matthews

Department of Psychology,

University of Warwick,

Coventry

CV4 7AL

UK

Tel: +44/0 2476523096

Fax: +44/0 2476524225

Email: w.j.matthews@warwick.ac.uk 


\begin{abstract}
In absolute identification experiments, the participant is asked to identify stimuli drawn from a small set of items which differ on a single physical dimension (e.g., ten tones which vary in frequency). Responses in these tasks show a striking pattern of sequential dependencies: The current response assimilates towards the immediately preceding stimulus but contrasts with the stimuli further back in the sequence. This pattern has been variously interpreted as resulting from confusion of items in memory, shifts in response criteria or the action of selective attention, and these interpretations have been incorporated into competing formal models of absolute identification performance. In two experiments, we demonstrate that lengthening the time between trials increases contrast to both the previous stimulus and the stimulus two trials back. This surprising pattern of results is difficult to reconcile with the idea that sequential dependencies result from memory confusion or from criterion shifts, but is consistent with an account which emphasizes selective attention.
\end{abstract}

KEYWORDS: Absolute identification; Inter-stimulus interval; Sequential effects; Assimilation; Contrast. 


\section{The effect of inter-stimulus interval on sequential effects in absolute identification}

On each trial of an absolute identification experiment, the participant is required to identify a stimulus drawn from a small set of items which differ on a single physical dimension (e.g., 10 tones which differ only in intensity). It has long been known that absolute identification data exhibit several interesting phenomena, including a severe limit in information transmission between stimuli and responses (e.g., Miller, 1956) and greater accuracy for stimuli at the ends of the stimulus range than in the middle (e.g., Murdock, 1960), and in the past few years there has been a resurgence of interest in absolute identification research (Brown, Marley, Donkin, \& Heathcote, 2008; Brown, Marley, \& Lacouture, 2007; Kent \& Lamberts, 2005; Petrov \& Anderson, 2005; Rouder, Morey, Cowan, \& Pfaltz, 2004; Stewart, 2007; Stewart, Brown, \& Chater, 2005).

A key result from absolute identification experiments is the finding that the response on the current trial $R_{n}$ to the presented stimulus $S_{n}$ depends upon the sequence of preceding stimuli. Typically, $R_{n}$ assimilates towards the immediately preceding stimulus $S_{n-1}$ but contrasts with (i.e., shifts away from) the stimuli presented two or more trials back $\left(S_{n-2}, S_{n-3} \ldots\right)$. The magnitudes of these effects, and the point in the sequence at which there is a switch from assimilation to contrast, depend upon the difficulty of the task and whether or not feedback is provided (e.g., Ward \& Lockhead, 1971), but the basic pattern has been found in a large number of studies (Holland \& Lockhead, 1968; Lacouture, 1997; Luce, Green, \& Weber, 1976, analysed in Jesteadt, Luce, \& Green, 1977; Staddon, King, \& Lockhead, 1980; Stewart et al., 2005; Treisman, 1985; Ward \& Lockhead, 1970; 1971). 
Over the past 40 years, this pattern of sequential effects has been given a number of different psychological interpretations, and these interpretations have been incorporated into competing formal models of absolute identification. Broadly speaking, the explanations for the sequential dependencies fall into three groups, which we now discuss in turn. Throughout, we focus on the core psychological ideas rather than detailed discussion of formal models, not least because recent models (e.g., Brown et al., 2008; Stewart et al., 2005) incorporate many free parameters and may possibly, with appropriate parameter choices, be rendered indistinguishable.

The first interpretation of sequential effects is that they represent the confusion of items in memory. This idea was first proposed by Holland and Lockhead (1968) and has appeared in various forms since then (Lockhead \& King, 1983; Lockhead, 1984; 1992). According to this interpretation, the representation of each stimulus is confused with the memories for earlier stimuli in the trial sequence. More recently, Stewart et al. (2005) have incorporated the idea of memory-confusion into a highly successful model which captures the full set of absolute identification choice data, including sequential effects. According to Stewart et al.'s relative judgment model (RJM), the participant estimates the difference between the current stimulus and the previous one (Laming, 1984). This difference is added to the feedback from the previous trial to produce a judgment of the current stimulus, with noise in the mapping of the perceived difference onto the response scale which leads to the information transmission limit seen in absolute identification. Crucially, the RJM assumes that the current stimulus difference is contaminated by residual representations of earlier stimulus differences, and that the more recently a stimulus difference occurred, the more likely it is to contaminate the judgment of the current difference. Stewart et al. (2005, pp. 896-897) show that, when feedback is provided and assuming that the participant's estimate of the stimulus difference corresponding to a single unit 
on the response scale is approximately correct, the confusion of stimulus differences predicts assimilation to $S_{n-1}$ and contrast to stimuli further back.

Memory-confusion interpretations of sequential effects like those of Stewart et al. (2005) suggest that the current response is predicted by the preceding stimulus sequence; preceding responses are ignored. The dependence of $R_{n}$ on the current and preceding stimuli may conveniently be assessed by fitting a regression equation to the data:

$$
R_{n}=r_{0}+\alpha_{0} S_{n}+\alpha_{1} S_{n-1}+\alpha_{2} S_{n-2}+\ldots+\alpha_{k} S_{n-k}+e_{n}
$$

Because only preceding stimuli are included as predictors in Equation 1, we refer to it as a stimulus-only regression. When Equation 1 is applied to absolute identification data, the coefficient for $S_{n-1}$ is positive, indicating assimilation, and the coefficients for stimuli further back in the sequence are negative, indicating contrast (e.g., Lockhead, 1984).

The second interpretation of sequential effects is that they represent trial-by-trial shifts in response criteria. A comprehensive statement of this idea is provided by criterion setting theory (Triesman \& Williams, 1984; Treisman, 1985). Criterion setting theory adopts a Thurstonian framework in which the presentation of each stimulus results in a noisy value on an internal sensory scale. Criteria, or response boundaries, divide the sensory scale into response categories and the response is determined by which criteria the (noisy) stimulus representation falls between. Each criterion has a long-term reference location, but the effective criteria on each trial are also influenced by two short-term processes: tracking and stabilization. The tracking mechanism is motivated by the idea that objects in the real world tend to persist, so that the presence of a particular object indicates that it is likely to occur again. Tracking therefore 
involves shifting the criteria away from the most recently made response, increasing the probability that the response will be repeated. Thus, the tracking mechanism produces assimilation to recent responses. The stabilizing mechanism, on the other hand, serves to place criteria near to the prevailing flux of sensory input. For example, if a series of sensory inputs all lie well above a criterion then that criterion may be too low in relation to the current distribution of inputs. Stabilization therefore shifts each criterion towards the current sensory input, producing contrast to recent stimuli.

Criterion setting theory thus asserts separate effects of preceding stimuli and preceding responses, with contrast to the former and assimilation to the latter. These effects suggest that absolute identification data may usefully be described with a regression equation in which both stimuli and responses are included as predictors:

$$
R_{n}=r_{0}+\alpha_{0} S_{n}+\alpha_{1} S_{n-1}+\beta_{1} R_{n-1}+\ldots+\alpha_{k} S_{n-k}+\beta_{k} R_{n-k}+e_{n}
$$

Because both preceding stimuli and preceding responses are included as predictors, we refer to Equation 2 as a stimulus-response regression. (Note that the term stimulus-response regression should not be taken to imply that the effects of preceding stimuli and responses are linked, only that both factors are being considered as predictors of the current response.) Mori and Ward (1995) applied a version of Equation 2 to absolute identification data and found that, in keeping with criterion setting theory, the coefficient for $R_{n-1}$ was positive but that the coefficient for $S_{n-1}$ was often negative, most notably in the absence of feedback - although the high correlation of stimuli and responses means that these coefficients may not be reliable. Mori (1998) similarly found assimilation to $R_{n-1}$ and contrast to $S_{n-1}$, although the latter changed to assimilation when 
the stimuli were masked. Both of these studies only considered the effects of the immediately preceding trial (Jesteadt et al., 1977).

The third interpretation of sequential effects is that they result from the operation of selective attention (e.g., Luce et al., 1976). Selective attention forms the basis of a recent, highly successful account of absolute identification - the selective attention, mapping, ballistic accumulator (SAMBA) model of Brown et al. (2008). SAMBA is able to predict the full range of choice and RT data from absolute identification experiments. At the heart of the model is a selective attention mechanism based on the rehearsal process of Marley and Cook $(1984 ; 1986)$. It is assumed that the stimulus dimension is monotonically mapped onto a linearly ordered set of leaky nodes. Throughout the experiment the participant rehearses a region of the stimulus dimension by directing rehearsal activity to a subset of these nodes. The upper and lower nodes in this range are referred to as anchors; their positions depend upon the range of stimuli in the experiment. To produce a magnitude estimate for the current stimulus the participant judges the position of the stimulus within the rehearsed range by calculating $\Sigma_{\mathrm{L}}$, the total rehearsal activation between the lower anchor and the current stimulus, and $\Sigma_{\mathrm{U}}$, the total activation between the stimulus and the upper anchor. The magnitude estimate is then $\Sigma_{\mathrm{L}} /\left(\Sigma_{\mathrm{L}}+\Sigma_{\mathrm{U}}\right)$. This magnitude estimate serves as input to Lacouture and Marley's (1995) mapping model, which produces a set of response strengths, one for each possible response. These response strengths serve as inputs to the final, decision stage of the model, which uses ballistic accumulators (Brown \& Heathcote, 2005) to produce a response. There is one ballistic accumulator corresponding to each possible response; each accumulator has a starting level of activation which then increases at a rate determined by the response strengths from the mapping stage. The first accumulator to reach a threshold level of activation determines the response and the time it 
takes to do so determines the RT.

Sequential effects are incorporated into SAMBA by the introduction of two additional assumptions. The first of these concerns the selective attention stage and comprises the idea that, on some trials, the participant directs rehearsal activity to the node corresponding to the most recently presented stimulus. For example, suppose the last stimulus was Stimulus 5. Rather than randomly allocating rehearsal activity between the lower and upper anchors, the participant directs the activity to the node corresponding to Stimulus 5. If the next stimulus lies above Stimulus 5, this redirected activity will contribute to $\Sigma_{\mathrm{L}}$ and the stimulus estimate will be larger than when the rehearsal activity is distributed randomly among the rehearsal nodes. Conversely, if the current stimulus lies below Stimulus 5 then the redirected activity will contribute to $\Sigma_{\mathrm{U}}$, resulting in a smaller magnitude estimate than normal. In this way, directing rehearsal activity to the location of the most recently presented stimulus causes subsequent responses to contrast with this stimulus. The second assumption concerns the decision stage of the model, in which ballistic accumulators race to threshold. Brown et al. (2008) assume that once an accumulator reaches threshold and a response is made, the activation begins to decay; the residual activity determines the starting point for each accumulator on the next trial. Since the accumulator with the greatest activity will be the one which reached threshold, the most recently made response will have a head start on the next trial, thereby increasing the probability that this response will be made again. Thus, the model predicts assimilation to the previous response. To summarize: SAMBA, like criterion setting theory, posits distinct effects of preceding stimuli and responses. Selective rehearsal of recent items produces contrast to the former whilst residual activity in the response system produces assimilation to the latter. 
It is clear from the foregoing survey that, while sequential effects provide a strong empirical constraint on formal models of absolute identification, the psychological interpretation of these effects remains disputed. In the current paper, we seek to clarify the interpretation of sequential dependencies by asking how the effects of previous stimuli and responses depend upon the time interval between trials. Manipulations of inter-stimulus interval have proved useful in distinguishing between interpretations of sequential effects in other tasks (e.g., Collier, 1954; DeCarlo, 1992) and several authors have recently argued for the importance of manipulating inter-stimulus interval in absolute identification (Brown et al., 2008; Lockhead, 2004; Stewart et al., 2005). The two experiments reported here represent the first attempt to do so.

\section{Experiment 1}

\section{Method}

Participants

Thirty nine members of the University of Warwick subject panel took part. Each was paid $£ 12$.

\section{Stimuli}

Ten tones of varying frequency were used. The lowest tone had a frequency of $600 \mathrm{~Hz}$ with each subsequent tone increasing in frequency by $12 \%$. Each tone had a total duration of $500 \mathrm{~ms}$ 
including a 50-ms amplitude ramp at beginning and end. Tones were played over Sennheiser eH2270 or HD265 headphones.

\section{Design and Procedure}

Participants were tested individually in quiet testing cubicles. Each participant completed two sessions, one with a short ISI and one with a long ISI. Each session consisted of four blocks of 90 trials; in each block, all ten tones were presented equally often with the order randomized for each participant. At the start of the experiment the ten tones were played in sequence from 1-10 with a 1s gap between each. While the tones played, the corresponding numbers appeared on the computer screen. Participants then began the experimental trials. On each trial participants heard one of the 10 tones; after the tone had finished, the participant indicated which of the tones had played by pressing one of the number keys along the top of a standard computer keyboard. In order to ensure a controlled interval between successive stimuli, participants were given a fixed temporal window in which to make their response. Previous studies have found that the vast majority of responses take less than $2 \mathrm{~s}$, so a $3 \mathrm{~s}$ window was used. At the end of the response window, the actual number of the presented tone was displayed for $500 \mathrm{~ms}$. The sequence of events on each trial was therefore: a 500ms 'Ready' signal, a 500ms blank interval, the stimulus for 500ms, a 3000ms response window, and feedback for 500ms. In the Short condition there followed a 500ms blank interval before the next trial; in the Long condition, this interval was $5500 \mathrm{~ms}$. Thus, in the Short condition the interval between the end of one stimulus and the start of the next was 3000 (response window) +500 (feedback) + 500 (ISI) + 500 ('Ready') + 500 
$($ blank $)=5000 \mathrm{~ms}$ whilst in the Long condition it was $3000+500+5500+500+500=$

10000ms. The experiment was controlled by DMDX (Forster \& Forster, 2003).

All participants had a break between sessions and some chose to come back on a different day to complete the second session. The first block of trials from each session was treated as practice and excluded from the analysis.

\section{Results and Discussion}

One participant was discarded because of technical difficulties; a second was discarded because of failing to respond within the 3 s response window on a large proportion (14.1\%) of trials (the mean proportion for the remaining subjects was $1.0 \%$ ). For two of the 37 useable participants, one block of trials was discarded because the test session was briefly interrupted; 18 participants completed the sessions in the order Short-Long and 19 in the order Long-Short.

\section{Basic Performance}

The average proportion of trials on which the participant failed to respond in the $3 \mathrm{~s}$ window in the Short condition was $0.7 \%(S D=1.0)$; the mean for the Long condition was $1.4 \%(S D=1.7)$. We used a mixed ANOVA to examine the effects of ISI and session order (Short-Long or LongShort) on the proportion of missed responses. There was a main effect of condition, $F(1,35)=$

$6.70, \eta_{p}^{2}=.16, p=.014$, with significantly more missed responses in the Long condition than the Short condition. (Here and throughout what follows, the criterion for significance was set at $\alpha=.05$ ). Although significant, this difference is very small and the very low number of missed 
responses in both conditions indicates that our choice of a $3 \mathrm{~s}$ response window was appropriate. There was no effect of session order, $F(1,35)<1$, and no interaction, $F(1,35)<1$. The mean proportion of correct responses (excluding missed responses) in the Short condition was 54.0\% $(S D=10.3)$, the mean for the Long condition was $52.7 \%(S D=10.5)$. Analysis of variance indicated no effect of condition, $F(1,35)=1.37, \eta_{p}^{2}=.04, p=.249$, no effect of session order, $F(1,35)<1$, and no interaction, $F(1,35)<1$.

In short, analysis of accuracy and proportion of missed trials shows that these statistics vary only slightly with changes in ISI and do not compromise the analysis of sequential effects which are the main focus of the current paper.

\section{Sequential Effects}

The effects of the preceding stimulus sequence on the current response are illustrated in Figure 1. This figure provides a so-called "impulse plot" (e.g., Lockhead, 1984). In an impulse plot, the mean error on the $n$th trial (averaged across all $S_{n}$ ) is shown as a function of the lag, $k$, for all possible $S_{n-k}$. The left panel shows the results from the Short ISI condition; the right panel shows the results from the Long ISI condition. (As is usual, the data have been collapsed over pairs of stimuli.)

Consider first the data from the Short condition. There is assimilation to the stimuli presented at lag $k=1$, because the mean error on trial $n$ is positive when the stimuli presented on trial $n-1$ are large, and negative when the stimuli presented on trial $n-1$ are small. However, the effect of stimuli shown at lag $k=2$ is the opposite; when $S_{n-2}$ was large, the mean error on trial $n$ was negative, and when $S_{n-2}$ was small the mean error was positive. The same is true of 
stimuli at longer lags. The left panel of Figure 1 therefore illustrates the classic pattern of assimilation to $S_{n-1}$ and contrast to $S_{n-2}$ seen in many previous studies of absolute identification (e.g., Lockhead, 1984; Stewart et al., 2005; Ward \& Lockhead, 1970; 1971) When we turn to the results from the Long ISI condition, however, two differences are apparent. Firstly, the assimilation to $S_{n-1}$ has disappeared and, if anything, been replaced by weak contrast. Secondly, the magnitude of the contrast to $S_{n-2}$, and possibly to stimuli further back in the sequence, has increased. Figure 1 therefore suggests that increasing the ISI has led to a general increase in contrast.

Impulse plots provide a convenient way to visualise the data, but they do not permit a quantitative analysis. Moreover, they do not separate the effects of preceding stimuli and preceding responses. We therefore fit Equations 1 and 2 to the data, to obtain a clearer understanding of the effects of inter-stimulus interval on sequential effects.

\section{Stimulus-only regression}

We began by fitting the stimulus-only regression equation (Equation 1) using stimuli up to five trials back in the sequence as predictors. (Responses which fell outside the $3 \mathrm{~s}$ response window were excluded from the analysis.) We conducted the regression analysis separately for both the Short and Long ISI conditions for each participant and then compared the regression coefficients from the two conditions. (An alternative approach would be to include the data from both conditions in a single regression which included ISI and the interactions between ISI and the various $S_{n-k}$ terms as predictors. The pattern of results from such an analysis are identical to those reported here.) The means and standard deviations of the regression coefficients for the 37 
participants are presented in the left of Table 1. Inspection of the regression coefficients reveals the same pattern as Figure 1. In the Short ISI condition, the coefficient for $S_{n-1}$ is moderately large and positive, indicating assimilation, whilst the coefficient for $S_{n-2}$ is negative, indicating contrast. In the Long ISI condition, the coefficient for $S_{n-1}$ has dropped to slightly below zero, whilst the coefficient for $S_{n-2}$ has become more negative. Finally, we note that the fit of the regression equation is good ( $R^{2}$ approximately .85), and little affected by the ISI condition. This is true of all of the regression equations fit in both experiments.

As one would expect when a large number of participants complete only a few hundred trials each, there was individual variation in the regression coefficients. We therefore used analysis of variance to statistically test the effects of ISI on the regression coefficients (Lorch \& Myers, 1990) ${ }^{1}$. We conducted a mixed ANOVA with ISI (Short vs. Long) as a within-subject factor and session order (Short-first vs. Long-first) as a between subjects factor. (Throughout what follows, the effects of session order were not significant unless otherwise stated.) The right side of Table 1 shows the ANOVA results for the main effect of ISI on each coefficient. The results show that inter-stimulus interval exerted a significant effect on the coefficients for $S_{n-1}$ and $S_{n-2}$; increasing the ISI significantly reduced assimilation to $S_{n-1}$ and significantly increased contrast to $S_{n-2}$. The coefficients for stimuli further back in the trial sequence were not significantly affected by the ISI manipulation.

\section{Stimulus-response regression}


To investigate the separate effects of preceding stimuli and responses, we applied Equation 2 to the data from Experiment 1. For this analysis, we considered the effects of stimuli and responses from the previous two trials. The mean and standard deviations of the regression coefficients are shown in the bottom of Table 1. Also shown at the right of the table are the ANOVA results for the main effect of ISI condition.

The coefficient for $S_{n-1}$ is significantly influenced by the inter-stimulus interval. In the Short condition, it is close to zero; in the Long condition, it is markedly negative, indicating a shift to contrast as the ISI is increased. A similar pattern is found for $S_{n-2}$, with contrast in the Short condition becoming significantly more pronounced in the Long condition (although the $p$ value for this effect is not particularly small; as a general point in the current work, we suggest that any significant results for which the $p$ value is not considerably less than .05 be treated with some caution). The effects of previous responses are not influenced by ISI. There is moderate assimilation to $R_{n-1}$ and very little effect of $R_{n-2}$, neither of which is influenced by the ISI manipulation. There was also a significant main effect of session order for the $R_{n-2}$ coefficient, $F(1,35)=5.20, p=.029, \eta_{p}^{2}=.13$, although session order did not interact with experimental condition, $F(1,35)=1.41, p=.243, \eta_{p}^{2}=.04$. This result is hard to interpret, and we do not consider it further.

In short, increasing the ISI makes the coefficients for both $S_{n-1}$ and $S_{n-2}$ more negative. That is, in the Long condition, there is stronger contrast to preceding stimuli than in the Short condition $^{2}$.

\section{Summary}


The results of Experiment 1 show that, irrespective of which regression model is used to assess the sequential effects, increasing the time between trials increases contrast to preceding stimuli. This is a novel and counter-intuitive result with strong theoretical implications, which we discuss below. Before that, we report a second experiment which sought both to establish the generality of this finding and to dissect the influence of intervals between specific pairs of trials.

\section{Experiment 2}

In Experiment 1, the Short and Long ISI conditions were grouped into blocks of trials. In Experiment 2, short and long inter-stimulus intervals were randomly intermixed throughout the experiment. The motivation for this was twofold. Firstly, we sought to replicate the striking pattern of results obtained in Experiment 1 under different conditions. Secondly, we sought to clarify which inter-stimulus intervals contribute to the observed effects. For example, in Experiment 1 it was found that increasing the ISI caused increased contrast to $S_{n-2}$. Was this because of the increased interval between $S_{n-2}$ and $S_{n-1}$, the increase between $S_{n-1}$ and $S_{n}$, or both? Randomly intermixing short and long inter-stimulus intervals throughout the trial sequence allows us to answer this type of question.

\section{Method}

\section{Participants}


Thirty eight members of the University of Warwick subject panel took part. Each was paid $£ 6$.

\section{Stimuli}

The stimuli were the same as Experiment 1.

\section{Design \& Procedure}

Each participant completed a single experimental session consisting of five blocks of 80 trials. In each block, each of the ten tones was presented eight times, four times followed by a short ISI and four times followed by a long ISI. The order was randomized for each participant.

At the start of the experiment, the ten tones were played in sequence from 1-10 with a $1 \mathrm{~s}$ gap between each. While the tones played, the corresponding numbers appeared on the computer screen. Participants then began the experimental trials. On each trial, participants heard one of the 10 tones; after the tone had finished, the participant had 2.5 seconds to indicate which of the tones had played by pressing one of the number keys along the top of a standard computer keyboard. At the end of the response window, the actual number of the presented tone was displayed for 500ms. On trials where participants failed to make a response, the words 'Too slow' were presented with the numerical feedback. Following the feedback there was a blank interval of 500ms (short ISI) or 5000ms (long ISI) after which the word 'Ready' was shown for $500 \mathrm{~ms}$ followed by a further $500 \mathrm{~ms}$ blank interval before the next tone played.

The total interval between the end of one tone and the start of the next was therefore 2500 $($ response window $)+500($ feedback $)+500($ ISI $)+500\left({ }^{(' R e a d y '}\right)+500($ blank $)=4500 \mathrm{~ms}$ in the 
short ISI condition and $2500+500+5000+500+500=9000 \mathrm{~ms}$ in the long ISI condition. The first block of trials from each session was treated as practice and excluded from the analyses.

\section{Results and Discussion}

Two participants failed to respond within the $2.5 \mathrm{~s}$ window on a large proportion of trials $(19.9 \%$ and $20.8 \%$ ) and were excluded from the analysis; the mean proportion of missed responses for the remaining participants was $1.5 \%$.

We refer to the interval between $S_{n-2}$ and $S_{n-1}$ as $I S I_{n-2, n-1}$, and to the interval between $S_{n-1}$ and $S_{n}$ as $I S I_{n-1, n}$. The data were organized according to $I S I_{n-2, n-1}$ and $I S I_{n-1, n}$. For each trial, the lengths of $I S I_{n-2, n-1}$ and $I S I_{n-1, n}$ were established and the data organized into the four possible combinations of the two ISIs: S,S (i.e., both ISIs were short: $I S I_{n-2, n-1}=4.5 \mathrm{~s}$ and $I S I_{n-1, n}$ $=4.5 \mathrm{~s}), \mathrm{S}, \mathrm{L}$ (a short ISI followed by a long ISI, $\left.I S I_{n-2, n-1}=4.5 \mathrm{~s}, I S I_{n-1, n}=9 \mathrm{~s}\right), \mathrm{L}, \mathrm{S}\left(I S I_{n-2, n-1}=\right.$ $\left.9 \mathrm{~s}, I S I_{n-1, n}=4.5 \mathrm{~s}\right)$ and L,L $\left(I S I_{n-2, n-1}=9 \mathrm{~s}, I S I_{n-1, n}=9 \mathrm{~s}\right)$. Note that we label the conditions to reflect, from left to right, the order in which the intervals were experienced. Thus condition $\mathrm{S}, \mathrm{L}$ corresponds to the stimulus sequence: $S_{n-2}$ - short interval - $S_{n-1}$ - long interval - $S_{n}$. In order to have a useable number of trials in each condition, the length of the ISI separating $S_{n-3}$ and $S_{n-2}$ was ignored, as were other ISIs further back in the sequence.

\section{Basic Performance}


The proportion of trials on which each participant failed to respond was calculated for each ISI condition. In condition $\mathrm{S}, \mathrm{S}$ the mean proportion was $1.0 \%(S D=1.5)$; in condition $\mathrm{S}, \mathrm{L}$ the mean was $1.6 \%(S D=2.3)$; in condition $\mathrm{L}, \mathrm{S}$ the mean was $1.2 \%(S D=1.5)$ and in condition $\mathrm{L}, \mathrm{L}$ the mean was $2.1 \%(S D=2.7)$. A $2 \times 2$ within-subject ANOVA revealed a marginally significant effect of $I S I_{n-2, n-1}, F(1,35)=4.13, p=.050, \eta_{p}^{2}=.11$, and a significant effect of $I S I_{n-1, n}, F(1,35)$ $=8.28, p=.007, \eta_{p}^{2}=.19$, but no interaction, $F(1,35)<1$. As in Experiment 1 , the overall proportion of missed responses was very low, indicating that the choice of response window (2.5s) was appropriate. The mean proportion of correct responses (excluding missed responses) was also calculated for each condition. In condition $\mathrm{S}, \mathrm{S}$, the mean was $54.1 \%(S D=14.9)$; in condition $\mathrm{S}, \mathrm{L}$ the mean was $52.1 \%(S D=12.6)$; in condition $\mathrm{L}, \mathrm{S}$ the mean was $53.2 \%(S D=$ 14.2); in condition $\mathrm{L}, \mathrm{L}$ the mean was $51.1 \%(S D=13.1)$. A $2 \times 2$ within-subjects ANOVA revealed no significant effect of $I S I_{n-2, n-1}, F(1,35)=1.16, p=.289, \eta_{p}^{2}=.03$, or $I S I_{n-1, n}, F(1,35)=$ $3.66, p=.064, \eta_{p}^{2}=.09$, and no interaction, $F(1,35)<1$.

\section{Sequential Effects}

As in Experiment 1, we begin by considering impulse plots for each of the four conditions. These are shown in Figure 2. The top left panel shows the results when both intervals were short. There is assimilation to $S_{n-1}$ and weak contrast to $S_{n-2}$. The bottom left panel shows the results when the interval between $S_{n-2}$ and $S_{n-1}$ was long and the interval between $S_{n-1}$ and $S_{n}$ was short.

The assimilation to $S_{n-1}$ has not changed, but the contrast to $S_{n-2}$ has become more pronounced. The top right panel shows the results when the interval between $S_{n-2}$ and $S_{n-1}$ was short but the 
interval between $S_{n-1}$ and $S_{n}$ was long. Now the assimilation to $S_{n-1}$ has been replaced by weak contrast; there is also contrast to $S_{n-2}$, and the magnitude of this effect is similar to that seen in condition L,S. Finally, the bottom right panel shows the results when both intervals are long. As in the preceding condition, there is weak contrast to $S_{n-1}$, and contrast to $S_{n-2}$ which is even stronger than before.

To quantify these findings, we again fit Equations 1 and 2 to the data.

\section{Stimulus-only regression}

For each of the four $I S I_{n-2, n-1}, I S I_{n-1, n}$ combinations, we fit a stimulus-only regression model (Equation 1) to the data from each participant. The mean and standard deviations of the regression coefficients for the 36 participants are presented in the upper portion of Table 2. (As in Experiment 1, an alternative approach is to conduct a single regression for the data from all four conditions with the inclusion of interaction terms to assess the effects of $I S I_{n-2, n-1}$ and $I S I_{n-1, n}$. The results are identical to those reported here.)

The coefficients were entered in a $2 \times 2$ within-subject ANOVA. The upper portion of Table 3 shows the $F$ values, $p$ values, and effect sizes for the main effects of $I S I_{n-2, n-1}$ and $I S I_{n-1, n}$ and for the interaction term. For the $S_{n}$ coefficient, there is a main effect of $I S I_{n-1, n}$ and an $I S I_{n-2, n-1} * I S I_{n-1, n}$ interaction. When $I S I_{n-2, n-1}$ is short, the coefficient is unaffected by $I S I_{n-1, n}$. When $I S I_{n-2, n-1}$ is long, the $S_{n}$ coefficient is larger when $I S I_{n-1, n}$ is long than when $I S I_{n-1, n}$ is short. Of more interest are the results for $S_{n-1}$ and $S_{n-2}$. The results confirm the 
pattern suggested by inspection of the impulse plots in Figure 3: There is a main effect of $I S I_{n-1, n}$ on the coefficient for $S_{n-1}$, but no effect of $I S I_{n-2, n-1}$ and no interaction. Both $I S I_{n-2, n-1}$ and $I S I_{n-1, n}$ significantly affect the coefficient for $S_{n-2}$, but again there is no interaction. The lack of interaction between the effects of $I S I_{n-2, n-1}$ and $I S I_{n-1, n}$ suggests that it is the total amount of time since $S_{n-2}$ was presented that determines the extent of contrast to this stimulus - i.e., the effects of $I S I_{n-2, n-1}$ and $I S I_{n-1, n}$ are additive.

\section{Stimulus-response regression}

We next applied a stimulus-response regression (Equation 2). The means and standard deviations of the regression coefficients are presented in the lower part of Table 2 . A series of $2 \times 2$ withinsubject ANOVAs were used to examine the effects of $I S I_{n-2, n-1}$ and $I S I_{n-1, n}$ on each coefficient and the results are shown in the lower half of Table 3. The pattern for $S_{n}$ is the same as for the stimulus-only regression. For the $S_{n-1}$ coefficient, there is a main effect of $I S I_{n-1, n}$; when the interval between $S_{n-1}$ and $S_{n}$ is short (i.e., in conditions S,S and L,S), there is weak assimilation to $S_{n-1}$, but when the interval is long the coefficient becomes negative, indicating contrast. There is no main effect of $I S I_{n-2, n-1}$ and no interaction. The coefficient for $R_{n-1}$ is moderately large and positive in all conditions; there is no effect of $I S I_{n-1, n}$, no effect of $I S I_{n-2, n-1}$, and no interaction. That is, assimilation to $R_{n-1}$ seems to be unaffected by inter-stimulus interval. Similarly, there is contrast to $S_{n-2}$ in all conditions. Inspection of the values for this coefficient suggests an increase in contrast as the time between $S_{n-2}$ and $S_{n}$ is increased. The coefficient is smallest in 
condition S,S and largest in condition L,L, with S,L and L,S having intermediate levels of contrast. However, the ANOVA indicates no effect of $I S I_{n-1, n}$, no effect of $I S I_{n-2, n-1}$, and no interaction. It seems likely that the effect of inter-stimulus interval on the coefficient for $S_{n-2}$ is no longer significant because of the increased noise in the regression parameters due to the multicollinearity among the stimulus and response predictors. (Recall also that the pattern of increasing contrast to $S_{n-2}$ seen here was found to be significant in Experiment 1, where there were more trials in each condition.) Finally, there is some evidence of weak assimilation to $R_{n-2}$, but as for $R_{n-1}$ there is no effect of $I S I_{n-1, n}$, no effect of $I S I_{n-2, n-1}$, and no interaction.

\section{Summary}

The results of Experiment 2 replicate those of Experiment 1. Both the stimulus-only and stimulus-response regression analyses indicate that increasing the time between trials increases contrast to preceding stimuli. In addition, the effect of $S_{n-1}$ depends only on $I S I_{n-1, n}$, whilst the effect of $S_{n-2}$ depends on both $I S I_{n-1, n}$ and $I S I_{n-2, n-1}$.

\section{General Discussion}

In both experiments, and irrespective of which regression equation was used to assess sequential effects ${ }^{3}$, increasing the time between trials led to increased contrast to preceding stimuli. In Experiment 1, trials were blocked by inter-stimulus interval. The Short (5s) ISI condition produced results typical of previous work: When the current response was considered as a 
function of the preceding stimuli (ignoring previous responses, Equation 1), there was assimilation to $S_{n-1}$ and contrast to stimuli further back in the sequence (e.g., Ward \& Lockhead, 1970). However, when the ISI was increased to 10 s the assimilation to $S_{n-1}$ disappeared whilst the contrast to $S_{n-2}$ became more marked. That is, for both $S_{n-1}$ and $S_{n-2}$ there was a shift towards contrast. When the effects of preceding responses were also considered (Equation 2), the Short ISI condition indicated very little effect of $S_{n-1}$ and contrast to $S_{n-2}$, and the Long ISI condition showed a significant increase in contrast to both $S_{n-1}$ and $S_{n-2}$. There was also evidence of assimilation to $R_{n-1}$ which was unaffected by the time between trials. Experiment 2 replicated these findings, and additionally found that the effects of $S_{n-1}$ and $S_{n-2}$ depend the total time since their occurrence.

As we described above, sequential effects have variously been interpreted as resulting from memory confusion, from shifts in Thurstonian response criteria, or from selective attention. The surprising pattern of results found here imposes an important constraint on the psychological interpretation of sequential effects and on the formal models of absolute identification which incorporate these ideas. In what follows, we discuss these interpretations in turn and ask whether each can accommodate the pattern of results found in the two experiments.

\section{Memory confusion}

As described in the Introduction, sequential effects have long been taken to indicate the confusion of items in memory (e.g. Holland \& Lockhead, 1968). The most successful formulation of this idea is Stewart et al.'s (2005) relative judgment model, in which each item is 
judged relative to the previous one but with the judgment of the current stimulus difference contaminated by memory for previous differences. Existing memory-based interpretations of sequential dependencies emphasize the effects of preceding stimuli and ignore previous responses; the impulse plots and stimulus-only regression analyses therefore provide the appropriate description of the data for appraising the memory-confusion account.

The results of these analyses are difficult to reconcile with a memory-based interpretation of sequential effects. If sequential effects reflect the influence of memories for previously encountered stimuli, we would expect that influence to decrease as the time since the presentation of those stimuli is lengthened, because the memory trace fades over time (e.g., Wicklegren, 1974). This is true irrespective of whether the sequential effects result from memory for actual stimuli or from memory of stimulus differences. Even if one assumes that forgetting occurs over items rather than in physical time (e.g., McGeoch, 1932), the best that can be expected is that increasing the ISI will make no difference to the effects of previous stimuli. In Experiments 1 and 2, increasing the time between trials decreased assimilation to $S_{n-1}$, consistent with the idea that the memory for that stimulus diminished. However, contrast to $S_{n-2}$ became more pronounced, not less.

It might be premature to abandon Stewart et al.'s (2005) highly successful account of absolute identification on the basis of the current results. However, we can see no straightforward memory-based interpretation of the finding that increasing the time between trials diminishes the effect of the more recently presented stimulus but increases the effect of the more distantly presented item. Furthermore, the situation is not improved by asserting separate effects of preceding stimuli and responses. The stimulus-response regressions show that increasing the ISI leads to greater contrast to both $S_{n-1}$ and $S_{n-2}$, and it is again difficult to see 
why lengthening the time since the establishment of a memory trace should increase the effect of that trace on the current response.

\section{Shifts in response criteria}

The current data are also hard to reconcile with the idea that sequential effects result from shifts in Thurstonian response criteria. According to criterion setting theory (Treisman \& Williams, 1984), each trial establishes a tracking trace which shifts the response criteria away from the current response, and a stabilization trace which produces a smaller shift towards the current stimulus. These traces decay over time so that the criteria move back to their long-term reference locations. Criterion setting theory therefore predicts separate effects of recent stimuli and responses, with contrast to the former and assimilation to the latter. Increasing the time between trials should reduce both the assimilation to preceding responses and the contrast to preceding stimuli, and the stimulus-response regression analyses provide an appropriate description of the data against which to test these predictions.

The results of these analyses contradict criterion setting theory. In keeping with the model, we found assimilation to $R_{n-1}$; the magnitude of this effect was not affected by the ISI, but this may simply reflect a slow decay in the tracking trace. However, we also found that increasing the time between trials rendered the regression coefficients for $S_{n-1}$ and $S_{n-2}$ significantly more negative. This is exactly the opposite of what is predicted; criterion setting theory assumes that the stabilizing shifts decay linearly over time, such that increasing the ISI must decrease the magnitude of the contrast effect. 
Criterion setting theory is, of course, only one instantiation of the idea that sequential effects result from shifts in response criteria. Alternative criterion-setting models could be developed to account for the current data if one were willing to assume arbitrary shifts in response criteria. Specifically, one would need to postulate shifts which initially increased over time (to explain the increase in contrast with increasing inter-stimulus interval) but which eventually changed direction and began to decay (because it makes no sense for the criteria to drift apart indefinitely). It would be hard to motivate such assumptions: Whereas Treisman and Williams (1984) convincingly argue that the shifts in criteria predicted by criterion setting theory tune the response system to the prevalent flux of sensory information, it is difficult to see how the pattern of criterion shifts needed to explain the current data could be justified.

\section{Selective attention}

Our results are hard to interpret in terms of memory processes or shifts in response criteria. They are, however, consistent with an account based on selective attention, the SAMBA model of Brown et al. (2008). Like criterion setting theory, this account posits separate influences of preceding stimuli and responses such that a stimulus-response regression provides the most appropriate description of sequential effects, and, as we have shown, the results of such analysis show that increasing the time between trials produces an increase in contrast to both $S_{n-1}$ and $S_{n-2}$. This is the pattern of results predicted by Brown et al.'s model.

Recall that the key idea of SAMBA is that the participant selectively attends to the region of the stimulus dimension occupied by the experimental stimuli. This selective attention is modelled by a Poisson rehearsal process: The stimulus dimension is represented by an ordered 
series of nodes and the participant directs rehearsal activity to the nodes covering the range of stimuli presented during the experiment (Marley \& Cook, 1984). Increments in the activity of each node are offset by a passive decay process, and the magnitude of a given stimulus is judged by determining the proportion of total rehearsal activity which lies below (or above) the corresponding node. On some proportion of trials, the rehearsal activity is preferentially directed towards the most recently presented stimulus; this preferential rehearsal may also be considered a form of selective attention. If a subsequent stimulus lies above the preferentially-rehearsed node, the stimulus magnitude will be overestimated; if it lies below, the magnitude will be underestimated. Thus, preferential rehearsal of recent stimuli produces contrast to those stimuli.

This model predicts that contrast will increase with increasing ISI. Immediately after the presentation of $S_{n-1}$, the participant begins to direct rehearsal activity to the corresponding node. Meanwhile, the activity in all the other nodes passively decays. The longer this goes on, the greater the proportion of total rehearsal activity accruing to the $S_{n-1}$ node and, correspondingly, the greater the contrast effect. There will also be increased contrast to $S_{n-2}$, provided the model parameters are chosen such that the increased rehearsal of the $S_{n-2}$ node during the interval between $S_{n-2}$ and $S_{n-1}$ is not offset by increased decay during the interval between $S_{n-1}$ and $S_{n}$ (or if, as Brown et al., 2008, suggest, the redirection of rehearsal activity continues for more than one trial.) The selective attention mechanism embodied in Brown et al.'s SAMBA model therefore successfully predicts the key pattern of results found in the current experiments.

According to SAMBA, the magnitude estimate produced by the selective attention stage is fed into Lacouture and Marley's (1995) mapping model, the outputs of which serve as inputs to ballistic accumulators (Brown \& Heathcote, 2005) which produce the final response. Residual activation in the ballistic accumulators is responsible for assimilation to previous responses, and 
this effect is predicted to decrease over time. The current experiments found the predicted response assimilation, and although we did not observe a decline in this effect when the ISI was increased this may simply reflect a slow decay in ballistic accumulator activity. Crucially, the selective attention mechanism is not tied to the other elements of SAMBA. The selective attention component of SAMBA represents a general idea which may be incorporated into other psychophysical models, including ones which provide different explanations for response assimilation.

The selective attention component of SAMBA provides the only account of sequential effects which correctly predicts that increasing the ISI will increase contrast to preceding stimuli. However, there is one aspect of the current data which is potentially problematic for this account, namely that the stimulus-response regression analyses provide little evidence for contrast to $S_{n-1}$ in the short ISI conditions. In Experiment 1, the mean coefficient for $S_{n-1}$ in the Short condition was 0.009 (Table 1). In Experiment 2, the stimulus-response regression produced mean coefficients for $S_{n-1}$ of 0.030 in the S,S condition and 0.029 in the L,S condition (see the lower half of Table 2). One sample $t$-tests established that none of these coefficients are significantly different from zero. Mori and Ward (1995) similarly found that the coefficients for $S_{n-1}$ were sometimes positive, although they are typically strongly negative in the absence of feedback (see also Mori, 1998). We do not regard this as a serious problem because the selective attention mechanism is only expected to produce weak contrast to $S_{n-1}$ when the ISI is short, and the coefficients obtained from the stimulus-response regression used to identify stimulus-specific effects are noisy because of the multicollinearity among stimuli and response predictor variables. However, should future experiments find significant assimilation to $S_{n-1}$ (when $R_{n-1}$ is included in the regression model), the selective attention explanation will need to be modified. 
The current results have applicability beyond absolute identification. DeCarlo (1992) varied the ISI in a magnitude estimation experiment and found that, when a stimulus-response regression was used, there was contrast to $S_{n-1}$ which became more pronounced as the ISI increased. DeCarlo suggested that this pattern indicated a mis-specification of the regression model. However, the selective attention component of SAMBA produces a magnitude estimate and Marley and Cook (1986) developed a model for magnitude estimation based upon this mechanism but without the redirection of rehearsal activity which produces contrast. If the redirection of rehearsal activity to recently presented stimuli assumed by SAMBA also occurs in magnitude estimation experiments, then this might provide an explanation for DeCarlo's result. That is, in magnitude estimation, as in absolute identification, preferential rehearsal of recent items may produce contrast to preceding stimuli, the magnitude of which increases with increasing time between trials. Similarly, the current results argue against the idea that sequential effects result from confusion of items in memory or shifts in response criteria. Both of these ideas have been invoked as explanations for sequential effects in other psychophysical tasks (e.g., Lockhead, 1992; 2004; Treisman, 1984; Treisman \& Williams, 1984); their failure to capture the pattern in absolute identification casts doubt on their applicability in these situations, too.

One outstanding question concerns the reason for redirecting rehearsal activity to the most recently presented stimuli. This redirection produces contrast and also underlies the increase in contrast which results from increasing the ISI. Since contrast shifts the participant's response away from the correct value, we might ask what function the preferential rehearsal serves. In the real-world, recently presented stimuli are likely to occur again in the near future (Treisman, 1985). It may be that selectively attending to the most recent stimuli reduces the 
perceptual noise in the representation of these items, thereby improving the accuracy with which likely future stimuli are represented. In absolute identification tasks, perceptual noise is usually so small as to be irrelevant, but under other conditions the redirected rehearsal activity may improve performance. Alternatively, the preferential rehearsal of recent items may serve as a general tracking mechanism such that, if the range or distribution of stimuli suddenly change (e.g., Ward, 1987), the system reallocates rehearsal activity to the new values. 


\section{Acknowledgments}

This work was supported Economic and Social Research Council grant RES-000-23-1372. We thank Donald Laming and an anonymous reviewer for their incisive comments on an earlier version of this article. 


\section{Footnotes}

${ }^{1}$ When separate regression analyses are conducted for a number of different participants, it is common to use inferential statistics, such as $t$-tests and ANOVA, to test whether the mean coefficients differ from zero or differ between conditions (Lorch \& Myers, 1990). However, an alternative, arguably superior, approach is provided by multi-level analysis (Raudenbush \& Bryk, 2002). For all of the analyses reported here we conducted corresponding multilevel analyses and found the same pattern of significant results.

${ }^{2}$ A reviewer asked whether the results for the subject averaged data reflected the findings from individual participants. In the stimulus-only regression, the $S_{n-1}$ coefficient decreased when the ISI was lengthened for 27 of the 37 participants ( $p=.008$, two-tailed Binomial test) and the $S_{n-2}$ coefficient decreased for 25 of the 37 participants $(p=.047)$. The coefficients for the $S_{n}, S_{n-3}$, $S_{n-4}$ and $S_{n-5}$ terms decreased for 17, 15, 22 and 18 participants, respectively (all $p s>.3$ ). For the stimulus-response regression, increasing the ISI led to a decrease in the $S_{n-1}$ coefficient for 26 of the 37 participants $(p=.02)$. Similarly, 26 participants showed a decrease in the coefficient for $S_{n-2}$. The coefficients for $S_{n}, R_{n-1}$ and $R_{n-2}$ decreased for 15,17 and 15 participants, respectively (all $p$ s > 0.3). These results match those of the averaged data.

${ }^{3}$ In addition to Equations 1 and 2, several alternative regression equations for the assessment of sequential effects have been proposed (Lockhead 1984; Lockhead \& King, 1983; DeCarlo \& Cross, 1990; in addition, a reviewer suggested regressing the current response only on the 
preceding sequence of responses). These approaches produced the essentially the same results as those reported here. 


\section{References}

Brown, S., and Heathcote, A. (2005). A ballistic model of choice response time. Psychological Review, 112, 117-128.

Brown, S. D., Marley, A. A. J., Donkin, C., and Heathcote, A. (2008). An integrated model of choices and responses times in absolute identification. Psychological Review, 115, 396-425.

Brown, S., Marley, A. A. J., and Lacouture, Y. (2007). Is absolute identification always relative? Comment on Stewart, Brown, and Chater (2005). Psychological Review, 114, 528-532.

Collier, G. (1954). Intertrial association at the visual threshold as a function of intertrial interval. Journal of Experimental Psychology, 48, 330-334.

DeCarlo, L. T. (1992). Intertrial interval and sequential effects in magnitude scaling. Journal of Experimental Psychology: Human Perception and Performance, 18, 10801088.

DeCarlo, L. T., and Cross, D. V. (1990). Sequential effects in magnitude scaling: models and theory. Journal of Experimental Psychology: General, 119, 375-396. 
Forster, K. I., and Forster, J. C. (2003). DMDX: A Windows display program with millisecond accuracy. Behavior Research Methods, Instruments \& Computers, 35, $116-124$.

Holland, M. K., and Lockhead, G. R. (1968). Sequential effects in absolute judgments of loudness. Perception \& Psychophysics, 3, 409-414.

Jesteadt, W., Luce, R. D., and Green, D. M. (1977). Sequential effects in judgments of loudness. Journal of Experimental Psychology: Human Perception and Performance, 3, 92-104.

Kent, C., and Lamberts, K. (2005). An exemplar account of the bow and set-size effects in absolute identification. Journal of Experimental Psychology: Learning, Memory, and Cognition, 31, 289-305.

Lacouture, Y. (1997). Bow, range, and sequential effects in absolute identification: A response-time analysis. Psychological Research, 60, 121-133.

Lacouture, Y., and Marley, A. A. J. (1995). A mapping model of bow effects in absolute identification. Journal of Mathematical Psychology, 39, 383-395.

Laming, D. (1984). The relativity of 'absolute' judgments. British Journal of Mathematical and Statistical Psychology, 37, 152-183. 
Lockhead, G. R. (1984). Sequential predictors of choice in psychophysical tasks. In S. Kornblum \& J. Requin (Eds.), Preparatory states and processes (pp. 27-47). Hillsdale, NJ: Erlbaum.

Lockhead, G. R. (1992). Psychophysical scaling: judgments of attributes or objects? Behavioral and Brain Sciences, 15, 543-601.

Lockhead, G. R. (2004). Absolute judgments are relative: A reinterpretation of some psychophysical ideas. Review of General Psychology, 8, 265-272.

Lockhead, G. R., and King, M. C. (1983). A memory model of sequential effects in scaling tasks. Journal of Experimental Psychology: Human Perception and Performance, 9, 461-473.

Lorch, R. F., and Myers, J. L. (1990). Regression analyses of repeated measures data in cognitive research. Journal of Experimental Psychology: Learning, Memory, and Cognition, 16, 149-157.

Luce, R. D., Green, D. M., and Weber, D. L. (1976). Attention bands in absolute identification. Perception \& Psychophysics, 20, 49-54.

Marley, A. A. J., and Cook, V. T. (1984). A fixed rehearsal capacity interpretation of 
limits on absolute identification performance. British Journal of Mathematical and Statistical Psychology, 37, 136-151.

Marley, A. A. J., and Cook, V. T. (1986). A limited capacity rehearsal model for psychophysical judgments applied to magnitude estimation. Journal of Mathematical Psychology, 30, 339-390.

McGeoch, J. A. (1932). Forgetting and the law of disuse. Psychological Review, 39, 352-370.

Miller, G. A. (1956). The magical number seven, plus or minus two: some limits on our capacity for information processing. Psychological Review, 63, 81-97.

Mori, S. (1998). Effects of stimulus information and number of stimuli on sequential Dependencies in absolute identification. Canadian Journal of Experimental Psychology, 52, 72-83.

Mori, S., and Ward, L. M. (1995). Pure feedback effects in absolute identification. Perception \& Psychophysics, 57, 1065-1079.

Murdock, B. B. (1960). The distinctiveness of stimuli. Psychological Review, 67, 1631.

Petrov, A. A., and Anderson, J. R. (2005). The dynamics of scaling: A memory-based 
anchor model of category rating and absolute identification. Psychological Review, 112, $383-416$.

Raudenbush, S. W., and Bryk, A. S. (2002). Hierarchical linear models: applications and data analysis methods ( $2^{\text {nd }}$ ed.). Thousand Oaks, California: Sage Publications.

Rouder, J. N., Morey, R. D., Cowan, N., and Pfaltz, M. (2004). Learning in a unidimensional identification task. Psychonomic Bulletin \& Review, 11, 938-944.

Staddon, J. E. R., King, M., and Lockhead, G. R. (1980). On sequential effects in absolute judgment experiments. Journal of Experimental Psychology: Human Perception and Performance, 6, 290-301.

Stewart, N. (2007). Absolute identification is relative: A reply to Brown, Marley, and Lacouture (2007). Psychological Review, 114, 533-538.

Stewart, N., Brown, G. D. A., and Chater, N. (2005). Absolute identification by relative judgment. Psychological Review, 112, 881-911.

Treisman, M. (1984). A theory of criterion setting: An alternative to the attention band and response ratio hypotheses in magnitude estimation and cross-modality matching. Journal of Experimental Psychology, General, 113, 443-463/ 
Treisman, M. (1985). The magical number seven and some other features of category scaling: properties of a model for absolute judgment. Journal of Mathematical Psychology, 29, 175-230.

Treisman, M., and Williams, T. C. (1984). A theory of criterion setting with an application to sequential dependencies. Psychological Review, 91, 68-111.

Ward, L. M. (1987). Remembrance of sounds past: Memory and psychophysical scaling. Journal of Experimental Psychology: Human Perception and Performance, 13, 216-227.

Ward, L. M., and Lockhead, G. R. (1970). Sequential effects and memory in category judgments. Journal of Experimental Psychology, 84, 27-34.

Ward, L. M., and Lockhead, G. R. (1971). Response system processed in absolute judgment. Perception \& Psychophysics, 9, 73-78.

Wicklegren, W. A. (1974). Single-trace fragility theory of memory dynamics. Memory \& Cognition, 2, 775-780. 


\section{Tables}

Table 1. Regression coefficients for the stimulus-only regression (Equation 1) and stimulusresponse regression (Equation 2) from Experiment 1.

\begin{tabular}{|c|c|c|c|c|c|c|c|}
\hline \multirow{2}{*}{$\begin{array}{c}\text { Predictor or } \\
\qquad R^{2}\end{array}$} & \multicolumn{2}{|c|}{ Mean } & \multicolumn{2}{|c|}{$S D$} & \multirow[t]{2}{*}{$F(1,35)$} & \multirow[t]{2}{*}{$\eta_{p}^{2}$} & \multirow[t]{2}{*}{$p$} \\
\hline & Short & Long & Short & Long & & & \\
\hline \multicolumn{8}{|c|}{ Stimulus-only regression (Equation 1) } \\
\hline$S_{n}$ & 0.877 & 0.888 & 0.067 & 0.073 & 1.36 & .04 & .252 \\
\hline$S_{n-1}$ & 0.042 & -0.005 & 0.046 & 0.059 & 19.44 & .36 & $<.001$ \\
\hline$S_{n-2}$ & -0.037 & -0.059 & 0.029 & 0.030 & 14.40 & .29 & .001 \\
\hline$S_{n-3}$ & -0.034 & -0.032 & 0.025 & 0.031 & 0.09 & .00 & .765 \\
\hline$S_{n-4}$ & -0.021 & -0.022 & 0.029 & 0.022 & 0.01 & .00 & .930 \\
\hline$S_{n-5}$ & -0.014 & -0.015 & 0.027 & 0.030 & 0.00 & .00 & .991 \\
\hline$R^{2}$ & 0.855 & 0.860 & 0.072 & 0.071 & & & \\
\hline
\end{tabular}

Stimulus-response regression (Equation 2)

$\begin{array}{llllllll}S_{n} & 0.877 & 0.887 & 0.066 & 0.073 & 1.24 & .03 & .274 \\ S_{n-1} & 0.009 & -0.047 & 0.078 & 0.110 & 11.29 & .24 & .002 \\ R_{n-1} & 0.040 & 0.049 & 0.081 & 0.082 & 0.34 & .01 & .565 \\ S_{n-2} & -0.032 & -0.066 & 0.067 & 0.071 & 5.30 & .13 & .027 \\ R_{n-2} & -0.008 & 0.015 & 0.062 & 0.072 & 2.22 & .06 & .145 \\ R^{2} & 0.852 & 0.859 & 0.072 & 0.071 & & & \end{array}$


Table 2. Regression coefficients for the stimulus-only (Equation 1) and stimulus-response regression (Equation 2) analyses for Experiment 2.

\begin{tabular}{|c|c|c|c|c|c|c|c|c|}
\hline \multirow{2}{*}{$\begin{array}{l}\text { Predictor } \\
\text { or } R^{2}\end{array}$} & \multicolumn{4}{|c|}{ Mean } & \multicolumn{4}{|c|}{$S D$} \\
\hline & $\mathrm{S}, \mathrm{S}$ & $\mathrm{S}, \mathrm{L}$ & $\mathrm{L}, \mathrm{S}$ & $\mathrm{L}, \mathrm{L}$ & $\mathrm{S}, \mathrm{S}$ & $\mathrm{S}, \mathrm{L}$ & $\mathrm{L}, \mathrm{S}$ & $\mathrm{L}, \mathrm{L}$ \\
\hline \multicolumn{9}{|c|}{ Stimulus-only regression } \\
\hline$S_{n}$ & 0.880 & 0.887 & 0.859 & 0.907 & 0.074 & 0.088 & 0.082 & 0.066 \\
\hline$S_{n-1}$ & 0.068 & -0.010 & 0.080 & -0.011 & 0.069 & 0.076 & 0.068 & 0.066 \\
\hline$S_{n-2}$ & -0.015 & -0.038 & -0.025 & -0.064 & 0.057 & 0.048 & 0.047 & 0.054 \\
\hline$R^{2}$ & 0.852 & 0.831 & 0.846 & 0.857 & 0.081 & 0.097 & 0.084 & 0.081 \\
\hline
\end{tabular}

Stimulus-response regression

$\begin{array}{lllllllll}S_{n} & 0.885 & 0.890 & 0.864 & 0.908 & 0.074 & 0.088 & 0.079 & 0.068 \\ S_{n-1} & 0.030 & -0.048 & 0.029 & -0.080 & 0.113 & 0.155 & 0.128 & 0.141 \\ R_{n-1} & 0.043 & 0.046 & 0.059 & 0.079 & 0.107 & 0.148 & 0.115 & 0.133 \\ S_{n-2} & -0.033 & -0.042 & -0.048 & -0.077 & 0.109 & 0.111 & 0.119 & 0.116 \\ R_{n-2} & 0.016 & 0.000 & 0.037 & 0.015 & 0.121 & 0.123 & 0.120 & 0.107 \\ R^{2} & 0.861 & 0.836 & 0.857 & 0.863 & 0.074 & 0.094 & 0.076 & 0.082\end{array}$

Note. Column headings indicate $I S I_{n-2, n-1}, I S I_{n-1, n}$ durations: $\mathrm{S}=$ Short (4.5s), L = Long (9.0s) 
Table 3. ANOVA results for stimulus-only (Equation 1) and stimulus-response regression (Equation 2) coefficient from Experiment 2.

\begin{tabular}{|c|c|c|c|c|c|c|c|c|c|}
\hline \multirow[t]{2}{*}{ Predictor } & \multicolumn{3}{|c|}{$I S I_{n-2, n-1}$} & \multicolumn{3}{|c|}{$I S I_{n-1, n}$} & \multicolumn{3}{|c|}{$I S I_{n-2, n-1} * I S I_{n-1, n}$} \\
\hline & $F(1,35)$ & $\eta_{p}^{2}$ & $p$ & $F(1,35)$ & $\eta_{p}^{2}$ & $p$ & $F(1,35)$ & $\eta_{p}^{2}$ & $p$ \\
\hline \multicolumn{10}{|c|}{ Stimulus-only regression } \\
\hline$S_{n}$ & 0.00 & .00 & .965 & 15.68 & .31 & $<.001$ & 5.48 & .14 & .025 \\
\hline$S_{n-1}$ & 0.39 & .01 & .537 & 40.70 & .54 & $<.001$ & 0.60 & .02 & .445 \\
\hline$S_{n-2}$ & 4.65 & .12 & .038 & 14.31 & .29 & $<.001$ & 0.96 & .03 & .335 \\
\hline \multicolumn{10}{|c|}{ Stimulus-response regression } \\
\hline$S_{n}$ & 0.02 & .00 & .896 & 10.39 & .23 & .003 & 5.09 & .13 & .030 \\
\hline$S_{n-1}$ & 0.59 & .02 & .447 & 21.39 & .38 & $<.001$ & 0.63 & .02 & .431 \\
\hline$R_{n-1}$ & 1.44 & .04 & .239 & 0.39 & .01 & .535 & 0.19 & .01 & .662 \\
\hline$S_{n-2}$ & 1.44 & .04 & .239 & 1.01 & .03 & .321 & 0.31 & .01 & .583 \\
\hline$R_{n-2}$ & 0.98 & .03 & .329 & 0.86 & .02 & .360 & 0.04 & .00 & .853 \\
\hline
\end{tabular}




\section{Figure Legends}

Figure 1. Impulse plots for Experiment 1.

Figure 2. Impulse plots for Experiment 2. 
Figure 1.
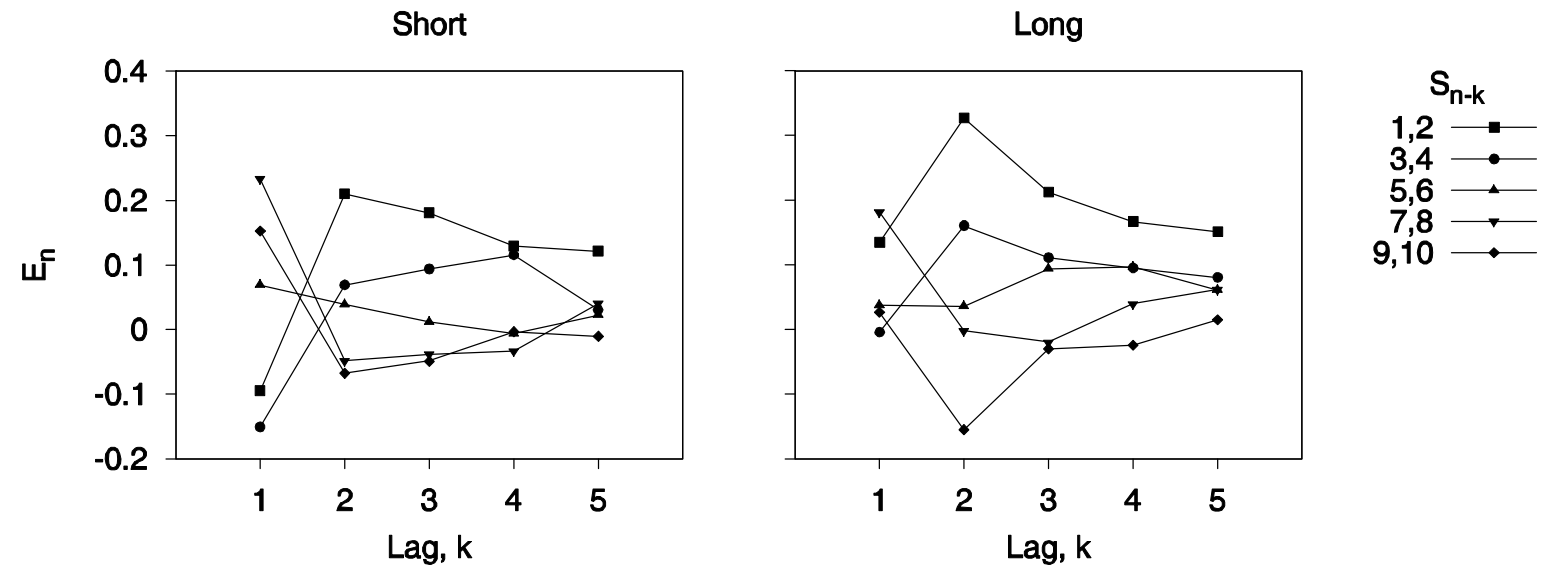
Figure 2.
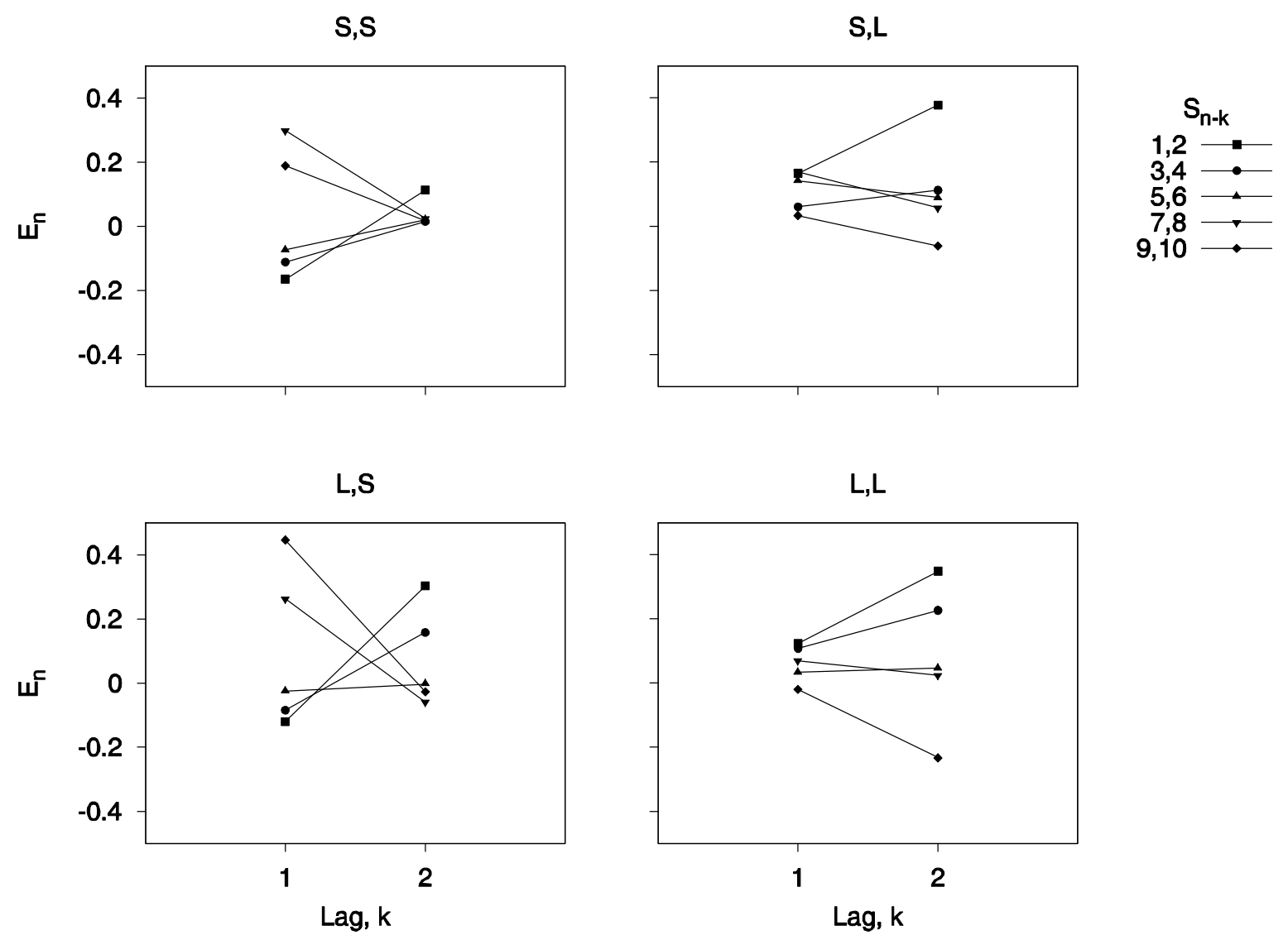

L,L

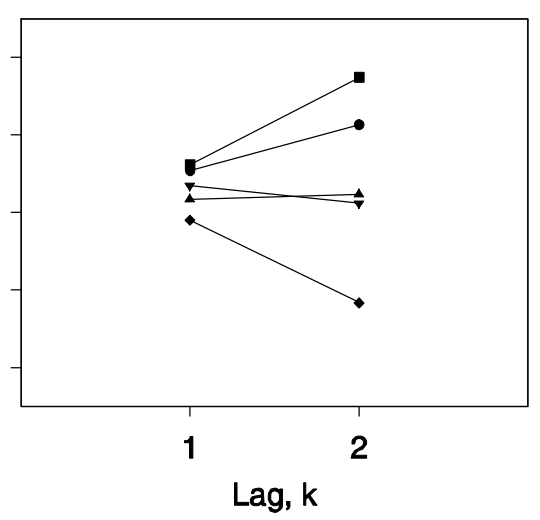

